\title{
An Advanced Nonlinear Signal Analysis Method For Damage Detection in Geomaterials
}

\author{
B. BaZARgan-Sabet,${ }^{1}$ H. Liu, ${ }^{1}$ and S. Chanchole ${ }^{1}$
}

\begin{abstract}
An algorithm is described which enables us to evaluate the Volterra kernels and the corresponding transfer functions. This method is then used to detect the cracking threshold of the geomaterials under loading. The responses of a sample of sandstone under axial leading and subjected to ultrasonic excitation are analyzed. The occurrence of microcracking is characterized by the changes in the linear and nonlinear parts of the measured signal energy.
\end{abstract}

Key words: Damage detection, signal processing, Volterra series, nonlinear analysis.

\section{Introduction}

Determining the cracking threshold in rock samples under compressive loading is very important for the study of rock behavior. Standard methods used for this purpose are essentially based on the measurement of the global strains (WOLTERS, 1971). However, this kind of method is not very sensitive. The density of cracks must reach relatively high levels before it produces measurable effects on deformations.

More sophisticated methods of detection use ultrasounds. Damage is thus detected by a modification in the characteristics of the output signal (GHOREYCHI, 1978). However, the results obtained by most of these methods are no more accurate than those obtained by the standard method of strain measurements. The reason is that signal analysis is generally restricted to the assumption of a linear system (governed by a linear convolution between the input signal and the impulse response). Since the process of microcracking is inelastic (GLADWIN and SACEY, 1974), linear processing can only provide partial information. In order to identify the damage threshold, it is therefore more appropriate to analyze the occurrence of nonlinearity in the output signal. Several possible methods exist for achieving this objective, among which the multidimensional treatment by the Volterra functional series seems to be particularly promising.

\footnotetext{
${ }^{1}$ Groupement pour l'étude des Structures souterraines de Stockage (G.3S), CNRS URA 317 (LMS)—Ecole Polytechnique, 91128 PALAISEAU Cedex, France.
} 
Although the Volterra series have been the subject of active research (BARRETT, 1963; ChOI and WARren, 1978; Hung et al., 1977) their use as part of a method of analysis for practical problems is still rare. Therefore we will begin by presenting an original algorithm which we have developed to separate the linear and nonlinear parts of the signal. We will then use this tool to detect the cracking threshold in geomaterials under compressive loading. Finally, we compare our results with those obtained by the standard global strain measurement method.

\section{Methodology}

Let $x(t)$ and $y(t)$ be respectively the input and output measurements for a dynamic system invariable in time. For example, the response $y(t)$ of a rock subjected to an ultrasonic excitation $x(t)$. The relationship between the two signals defined by the Volterra series is the following;

$$
y(t)=y_{1}(t)+y_{2}(t)+\cdots+y_{n}(t),
$$

where

$$
\begin{gathered}
y_{1}(t)=\int_{-\infty}^{\infty} h_{1}(\tau) x(t-\tau) d \tau, \\
y_{2}(t)=\int_{-\infty}^{+\infty} \int_{-\infty}^{+\infty} h_{2}\left(\tau_{1}, \tau_{2}\right) x\left(t-\tau_{1}\right) x\left(t-\tau_{2}\right) d \tau_{1} d \tau_{2}, \\
y_{n}(t)=\int_{-\infty}^{+\infty} \int_{-\infty}^{+\infty} h_{n}\left(\tau_{1}, \tau_{2}, \ldots, \tau_{n}\right) \prod_{i=1}^{n} x\left(t-\tau_{i}\right) d \tau_{i} .
\end{gathered}
$$

Here the first term $y_{1}(t)$ is a linear term. It is obtained by the convolution product between the first order kernel, $h_{1}(t)$, and the excitation function $x(t)$. The nonlinear behavior is evident from the presence of higher order terms $y_{2}(t), y_{3}(t), \ldots$ etc.

The number of possible terms in (1) is in theory infinite. In practice $n$ is chosen such that the difference between the recorded signal and the signal calculated based on the $n$ first components is reasonably small. This margin of error is fixed according to the equipment used for each application and the precision of the measurement.

The problem we wish to solve is the calculation of the kernels, given excitation $x(t)$ and output signal $y(t)$. To obtain large order kernels $h_{n}\left(t_{1}, t_{2}, \ldots, t_{n}\right)$, multidimensional deconvolutions are necessary, which are generally very difficult to achieve. However, in some cases it is possible to obtain solutions in which the input signal is assumed to be of a particular type. For example VINH (1987) uses excitation by shock (modeled by a Dirac distribution) which allows him to directly deconvolute the Volterra kernels of different orders. Others, like BoyD et al. (1983), use multi-harmonic excitations. 
In the case of ultrasounds, the input signal has a complex form. Indeed current transducer technology does not allow the generation of signals assimilable to Dirac distributions or of sufficiently pure harmonic waves. Here we propose a method for calculating Volterra kernels regardless of the form of the input signal. To do this we first express equation (1) in the frequency domain. Using the multidimensional Fourier transform and by extending Planchenel's theorem to dimensions greater than one, equation (1) can be written as:

$$
Y(f)=\sum_{i=1}^{n} Y_{i}(f)=\sum_{i=1}^{n} H_{1}\left(f_{1}, f_{2}, \ldots, f_{i}\right) \prod_{k=1}^{i} X\left(f_{k}\right) \quad\left(f \in f_{1}, f_{2}, \ldots, f_{n}\right),
$$

where $H_{i}\left(f_{1}, \ldots, f_{n}\right)$ is the Fourier transform of $h_{i}\left(t_{1}, \ldots, t_{n}\right)$. We must therefore calculate different order transfer functions $H_{1}(f), H_{2}\left(f_{1}, f_{2}\right), \ldots$, by solving the equation (5). To illustrate the resolution procedure, let us examine the case where $n=2$ :

$$
Y(f)=H_{1}\left(f_{1}\right) X\left(f_{1}\right)+H_{2}\left(f_{1}, f_{2}\right) X\left(f_{2}\right) X\left(f_{1}\right)
$$

or

$$
H_{2}\left(f_{1}, f_{2}\right)=\frac{Y(f)-H_{1}\left(f_{1}\right) X\left(f_{1}\right)}{X\left(f_{1}\right) X\left(f_{2}\right)} .
$$

To obtain $H_{2}\left(f_{1}, f_{2}\right)$ it is necessary to know the system's response in the plane $\left(f_{1}, f_{2}\right)$. To achieve this we return to the time domain and artificially reconstitute this response from the measurements of the signals $x(t)$ and $y(t)$. Figure 1 shows this reconstruction procedure. By shifting $k$ times the excitation $x(t)$ in time, we obtain $k$ new input signals $x_{i}(t)$ such as:

$$
x_{i}(t)=x(t)+x(t-i \Delta t) \quad i=1,2, \ldots, k
$$

$k \in \mathbf{N}$ defined by the length of the output signal.

For each $x_{i}(t)$, the corresponding response is placed parallel to the time axis $t$ in the plane $\left(t_{1}, t_{2}\right)$ with a shift $\Delta t$. We thus obtain $y\left(t_{1}, t_{2}\right)\left(t_{1} \geq 0, t_{2} \geq 0\right)$. The bidimensional Fourier transform of $y\left(t_{1}, t_{2}\right)$ provides the system response in the frequency domain, thereby allowing us to solve (7). By reproducing the construction procedure in space $\left(t_{1}, t_{2}, t_{3}\right)$ it is possible to extend the method to $n=3$ and thereafter to $n>3$.

However, for real applications it is often sufficient to take the case in which the frequency variables in (5) are identical $\left(f_{1} \equiv f_{2} \equiv \cdots \equiv f_{n} \equiv f\right)$. In other words, we reduce these multivariable functions to functions with a single variable by selecting specific planes of intersection. For example for $H_{2}\left(f_{1}, f_{2}\right)$ taking $\left(f_{1} \equiv f_{2} \equiv f\right)$, the second order transfer function is a function situated along the diagonal in the plane $\left(f_{1}, f_{2}\right)$. In the same way, taking $\left(f_{1} \equiv f_{2} \equiv f_{n} \equiv f\right)$, the third-order transfer function will be situated along the trisectrice. The information derived from these functions is sufficiently significant to be used in the interpretation of real experiments. This 
information can be complemented by including the functions on the axes with only one variable (LIU, 1991) ( for example $f_{1} \equiv f$ and $f_{2} \equiv f_{3} \equiv 0$ for $H_{3}$ ).

In order to evaluate the transfer functions in the specific case where $f_{1} \equiv$ $f_{2} \equiv \cdots \equiv f_{n} \equiv f$, we must use $(n)$ different signals. In practice we use the same input signal with $(n)$ different levels of amplitude. For the $m$ th level of excitation the Fourier transform of the corresponding response is:

$$
Y_{(m)}(f)=\sum_{i=1}^{n} H_{i}(f) X_{(m)}^{i}(f), \quad m=1,2, \ldots, n .
$$

We put (9) in matrix form by taking $m=n$ (which means that the number of levels of excitation is equal to the number of terms included in the Volterra series).

$$
Y=X H
$$

where

$$
Y=\left\{Y_{(1)}(f) \quad Y_{(2)}(f) \quad \cdots \quad Y_{(n)}(f)\right\}^{T}
$$
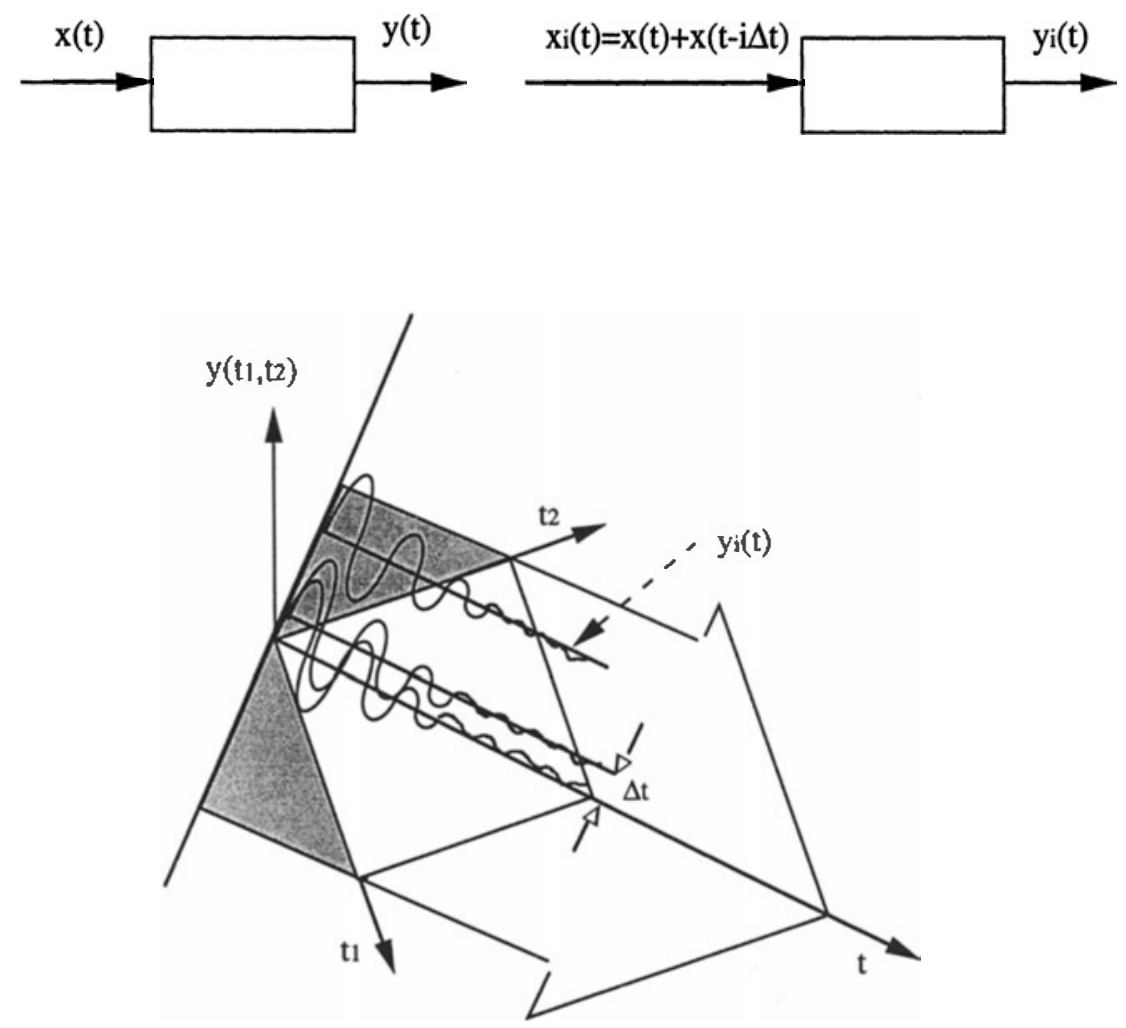

Figure 1

The reconstitution procedures for $y\left(t_{1}, t_{2}\right)$. 

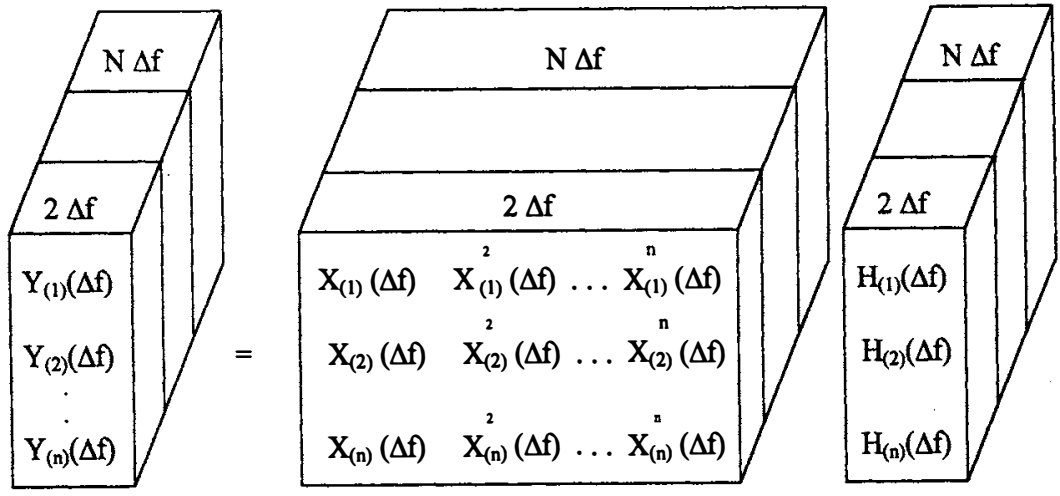

Figure 2

The process for solving equation (10).

$$
\begin{aligned}
& H=\left\{\begin{array}{llll}
H_{(1)}(f) & H_{(2)}(f) & \cdots & \left.H_{n}(f)\right\}^{T}
\end{array}\right. \\
& X=\left[\begin{array}{cccc}
X_{(1)}^{1}(f) & X_{(1)}^{2}(f) & \cdots & X_{(1)}^{n}(f) \\
X_{(2)}^{1}(f) & X_{(2)}^{2}(f) & \cdots & X_{(2)}^{n}(f) \\
\cdots & \cdots & \cdots & \cdots \\
X_{(n)}^{1}(f) & X_{(n)}^{2}(f) & \cdots & X_{(n)}^{n}(f)
\end{array}\right]
\end{aligned}
$$

Figure 2 shows the resolution process schematically.

Equation (10) is solved step by step up to the frequency $f_{\max }=N \Delta f(N$ is the number of samples) which must remain inferior or equal to the Nyquist frequency (defined by the sampling theorem).

\section{Practical Application}

Here we present the practical application of the described algorithm. We use it to detect the occurrence of microcracking in a sandstone sample during a uniaxial compressive test. In order to demonstrate the capacities of our method we have chosen to compare our results with those obtained by the standard global strain measurement method.

The following experiment is one of a group of 10 tests which we carried out on three different kinds of rock, as part of a research contract on radioactive waste storage. The results we obtained from each of these tests are all qualitatively similar. We therefore limit our detailed presentation to one representative test.

The sample studied is a cylinder $75 \mathrm{~mm}$ in diameter and $150 \mathrm{~mm}$ in height. We placed a broad band $(0.2 \mathrm{MHz}$ to $0.8 \mathrm{MHz})$ transducer on either side of the sample. 
The transmitter is connected to an ultrasound excitation generator, enabling the production of acoustic waves of between 0 and $5 \mathrm{kPa}$ of pressure. The axial loading is provided by a press of $1000 \mathrm{kN}$. The displacement sensors, positioned both axially and radially, allow calculation of the global strains during loading.

Axial stress as a function of axial strain shows very obvious elastic behavior, which is expressed by a linear relation between the axial load and the axial deformation, characteristic of this material (see Fig. 4). Figure 5 illustrates the volumic strain of the sample, reconstituted from the axial and radial deformations versus the axial strain. A deviation in the curve is clearly noticeable from a volumic strain of about $0.68 \%$, which corresponds to an axial load of $420 \mathrm{kN}$. The threshold of the appearance of cracking is generally situated at the point of this deviation (dilatation-contraction threshold) (BEREST et al., 1989).

We now examine the results obtained from signal analysis. Having separated the output signal into its linear and nonlinear components we will follow the evolution of each part as a function of load. As previously mentioned, the sample must be subjected to several levels of excitation. To ensure that nonlinearity obtained is due to the material and not to peripheral elements, we must use the lowest possible levels of input signal (HEITZ, 1992). For these experiments we selected three levels

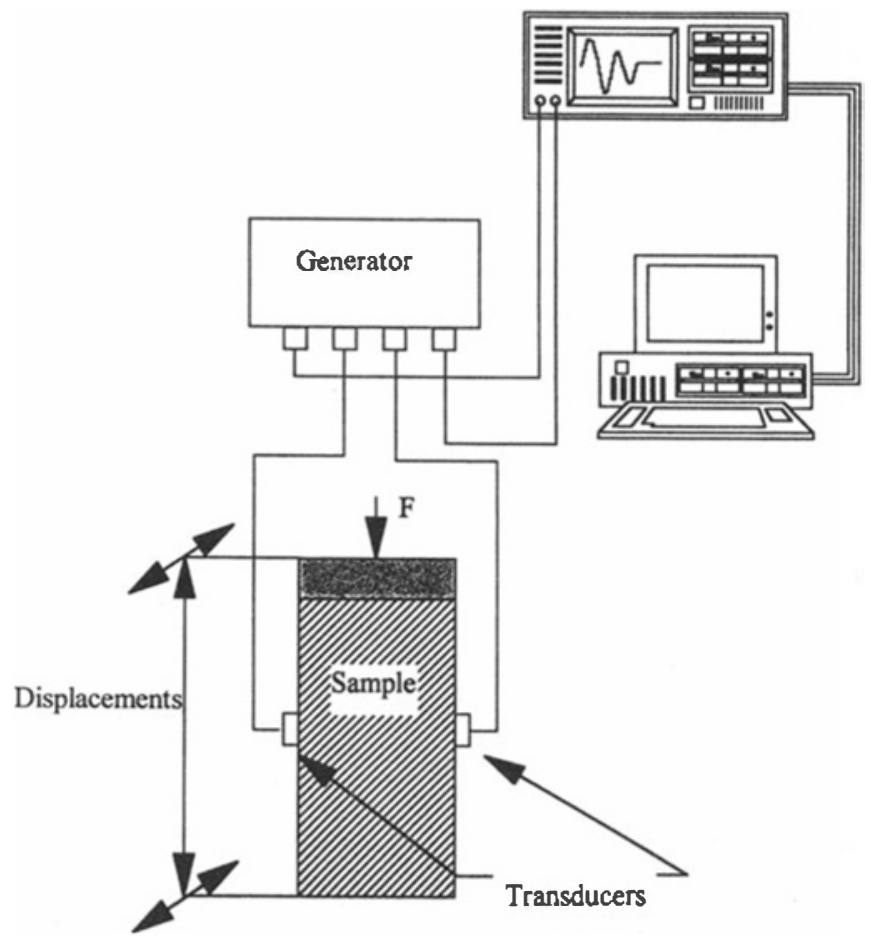

Figure 3

Experimental setting. 


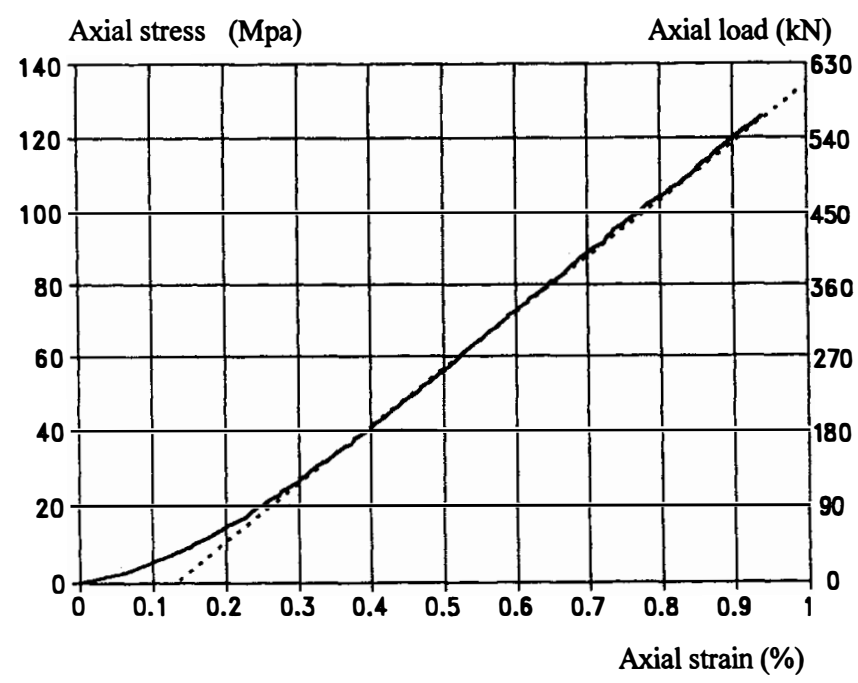

Figure 4

Axial stress versus axial strain.

equivalent to acoustic pressure of $0.25 \mathrm{kPa}, 0.5 \mathrm{kPa}$ and $1.0 \mathrm{kPa}$. In addition, we made sure that the transducers did not bear any loading. In this way the relative signal variations we recorded could not be attributed to the equipment.

Figure 6 shows the excitation signal in the time domain, recorded using a perfectly elastic reference material with properties similar to sandstone. Figure 7

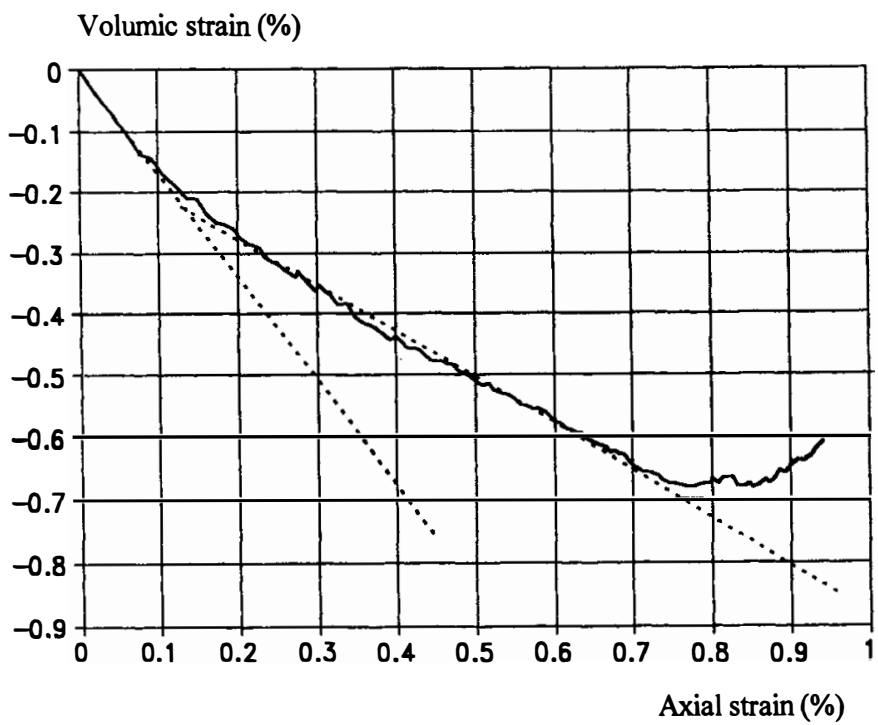

Figure 5

Volumic strain versus axial strain. 


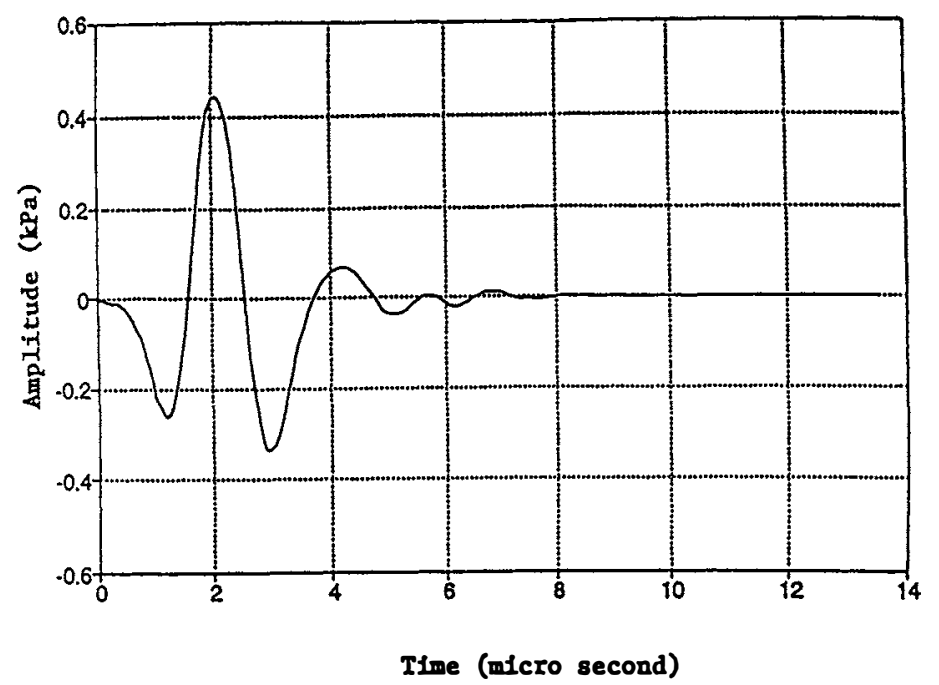

Figure 6

Excitation signal.

shows the evolution of sample responses $y(t$, load $)$ as recorded during loading. We fix the margin of error at $1 \%$ of the magnitude of the output signal. With three levels of excitation it is possible to calculate the first three Volterra kernels, so that:

$$
y(t)=y_{1}(t)+y_{2}(t)+y_{3}(t) .
$$

Our experience has shown that for most materials it is sufficient to consider three terms in the Volterra series. However, given the weak level of $y_{3}(t)$ (see Fig. 8) compared to the two other components $y_{1}(t)$ and $y_{2}(t)$, we may simply retain only the first two terms.
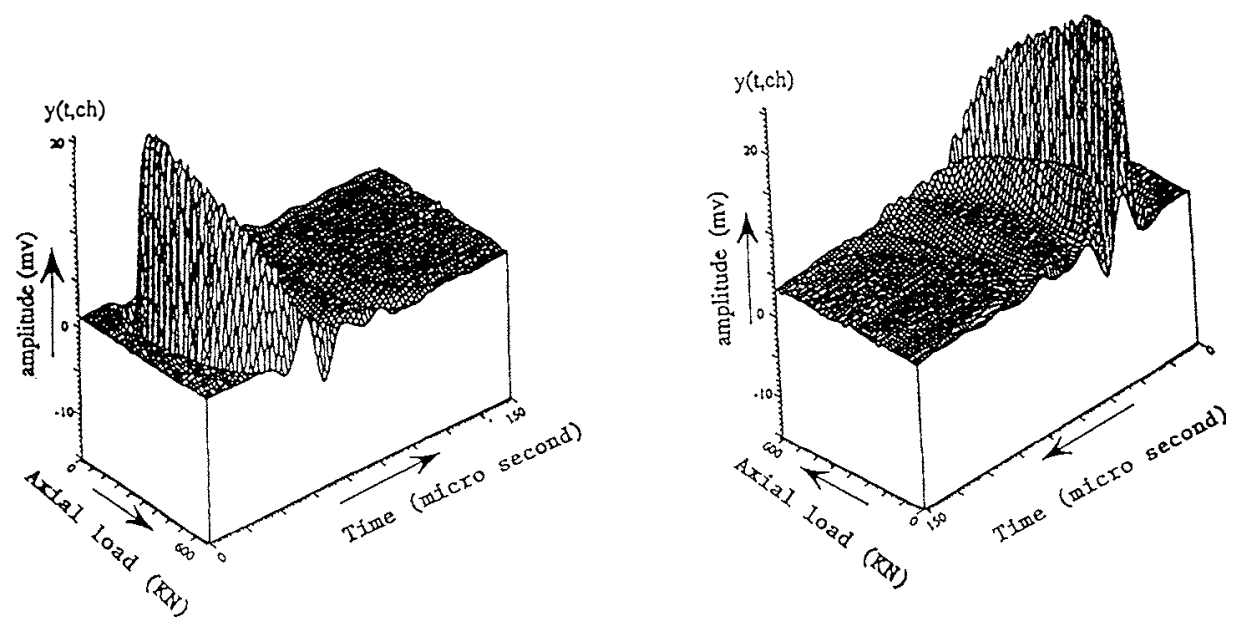

Figure 7

Sandstone responses according to axial deformation. 

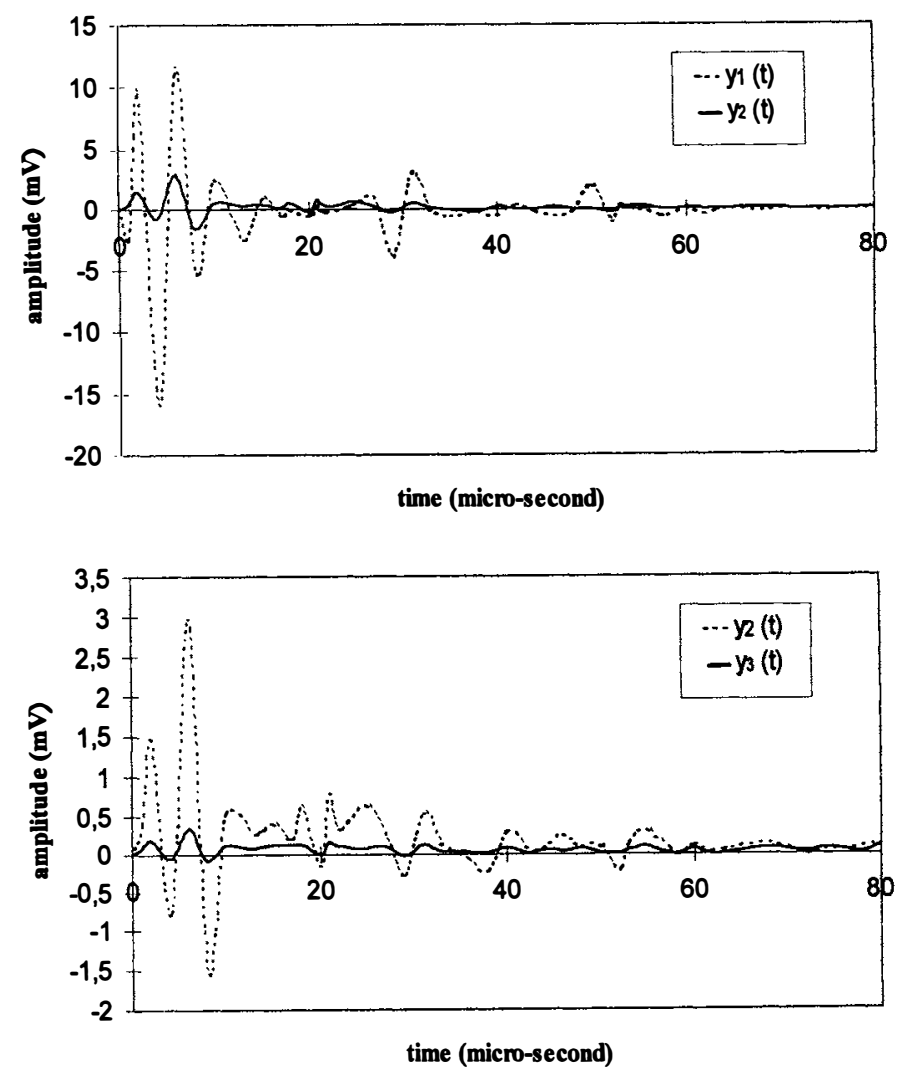

Figure 8

Comparison between the three components of the output signal $\left(y_{1}(t), y_{2}(t)\right.$ and $\left.y_{3}(t)\right)$ corresponding to $450 \mathrm{kN}$ load.

We take the ratio of the signal energy to the total energy transmitted as the adimensional parameter for which we will study the variations as a function of applied load. By "energy" we mean the quantity represented by:

$$
E\left(y_{k}(t)\right)=\int_{-\infty}^{\infty}\left|y_{k}(t)\right|^{2} d t .
$$

It would be possible to follow the variations of other signal parameters, for example the magnitude of the peak around the excitation's main frequency, but the energy parameter, which includes the variations across the entire frequency band, seems to be more appropriate.

Figure 9 shows the energy variation in the linear part of the signal $E\left(y_{1}\right)$ according to the axial load applied to the sample. This curve comprises three noteworthy parts: First, between 0 and $90 \mathrm{kN}$ which shows a fall in the value of $E\left(y_{1}\right)$. Then between 90 and $400 \mathrm{kN}$ showing stability in the value of $E\left(y_{1}\right)$. Finally from $400 \mathrm{kN}$ where we see a sharp rise in $E\left(y_{1}\right)$. 
Given the nature of the rock selected (sandstone behaves in an elastic-fragile manner), and the weak acoustic energy levels used, we may hypothesize that the system's behavior depends solely on the stiffness of the material. In other words, we assume that the mass and the viscous damping coefficient of the sample are conservative before the failure point. In that case the first part of the curve (9) corresponds to a tightening in the sample's structure. In this phase the linear stiffness of the rock increases and the linear response $y_{1}(t)$ consequently diminishes thus keeping with the dynamic equation $\mathbf{K} y(t)=a x(t)$ in which $\mathbf{K}$ is the linear stiffness (BUI, 1993). The stabilization phase corresponds to the elastic response of the material. Finally, the diminution in stiffness, when the material is damaged, causes a rise in $y_{1}(t)$.

Figure 10 demonstrates the energy variation of the nonlinear part of the signal $E\left(y_{2}\right)$ versus the axial load. It is interesting to note the difference in magnitude between this curve and that of the linear part. Nonlinearity represents only a small part of the total response. Similar to the linear part, this curve is formed of three parts: Initially, a rapid fall at the beginning of the curve which indicates the closure of existing microcracks. Then, a quasi-stability in the loading zone of between 100 and $300 \mathrm{kN}$. Finally a progressive rise in $E\left(y_{2}\right)$ from $310 \mathrm{kN}$. We may presume that this variation in nonlinear energy indicates the beginning of microcracking in the sample, the only possible modification of the physical state under compressive loading.

It is interesting to note that this last change in slope occurs well before that observed in the previous curve concerning the linear part of the energy. Similarly we would point out that in the zone preceding the slope change (between 100 and $300 \mathrm{kN}$ ), the magnitude of nonlinear energy approaches zero.

Normalized energy $E\left(y_{1}\right)$

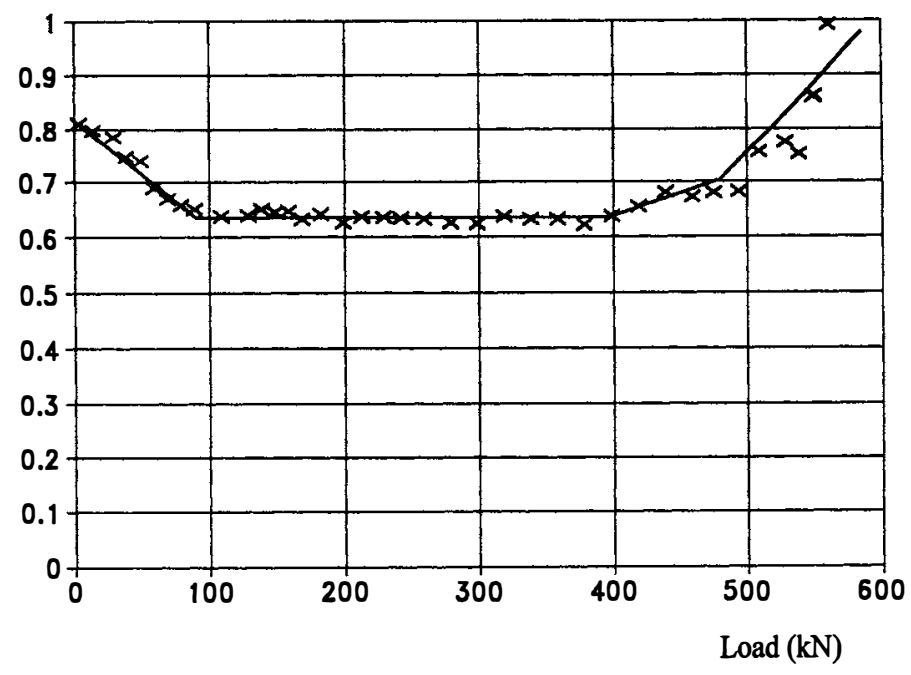

Figure 9

Signal energy variation of the first Volterra kernel versus the axial load. 


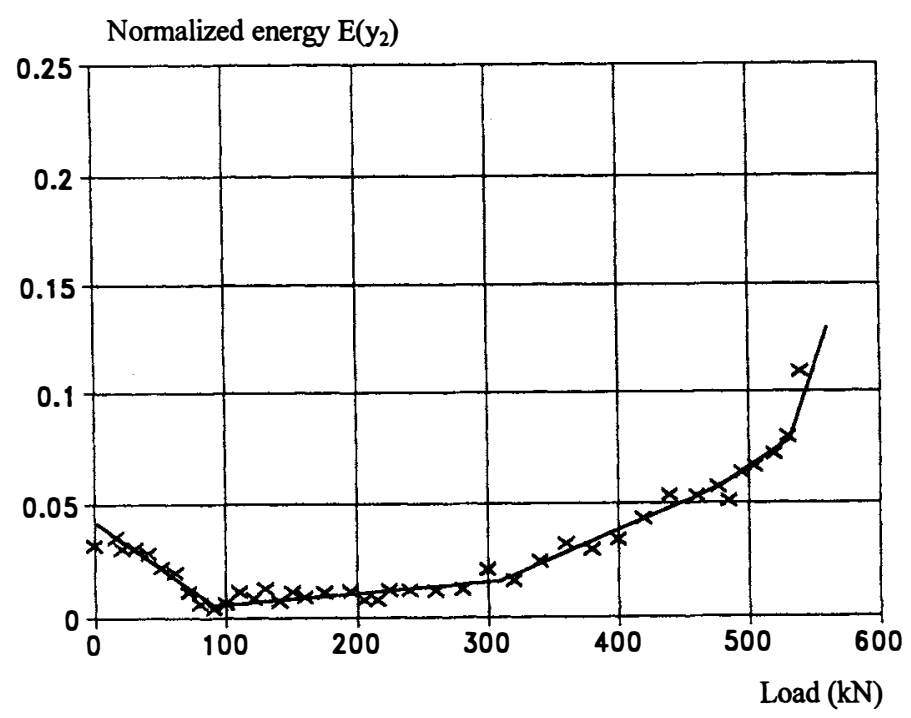

Figure 10

Signal energy variation in relation to the second Volterra kernel versus the axial load.

We now compare our results with those obtained by the standard method (curves in Figs. 4 and 5):

a. The initial closure of the microcracks and the tightening of the material are equally evident in the curves showing the volumic strain and the axial stress versus axial strain (slope change). Reference to the load/strain curve reveals that the end of this initial phase occurs around a stress of $20 \mathrm{MPa}$, i.e., an axial load of $90 \mathrm{kN}$. This corresponds to the beginning of the stabilization phase for both the linear and nonlinear energy variation curves.

b. The sample's dilation-contraction threshold shown on the volumic strain curve corresponds to an axial load of approximately $420 \mathrm{kN}$, equivalent to the value at which we observe a rapid increase in the linear part of the energy $E\left(y_{1}\right)$.

c. The material's behavior is purely elastic between 100 and $300 \mathrm{kN}$. This is evident in the curves, but is borne out by the fact that in this interval nonlinear energy is almost absent and linear energy remains constant (no variation in stiffness).

d. The rise in energy from a loading threshold of $310 \mathrm{kN}$ has no equivalent on the other curves. This change indicates structural modifications in the sample, probably related to the beginning of microcracking. Only when the density of the microcracks becomes sufficiently high to influence the material's stiffness ( from $400 \mathrm{kN}$ ), will the effect be visible in the other curves. 


\section{Other Results}

As mentioned, the above experiment is part of a research program. Output signals obtained from 9 other samples were also treated by the Volterra series. Three different kinds of rock were examined. Both uniaxial and triaxial (under confining pressure) compressive tests were carried out. The main results are summarized in Table 1.

As the results evidence, in all cases nonlinear analysis allows forward detection of damage. However, it is important to be aware that this method does not lead to a quantitative estimation of the damage level. Indeed, the growth of microcracks produces the rise of nonlinearity in the output signal, although there is no proportionality between crack density and the nonlinear part of the signal. Determining the quantity of microcracks necessitates further investigation and assumes a modeling of the rock dynamic behavior.

\section{Conclusion}

Signal analysis using the Volterra series appears to be an efficient tool for the detection of the microcracking threshold in geomaterials under compressive loading. As we have seen the occurrence of microcracks causes the appearance and subsequent increase of the nonlinear component of the Volterra series. Whereas the linear part of the signal produces comparable results to those obtained by the

Table 1

Identification of the damage threshold for three different rocks, using standard and nonlinear methods

\begin{tabular}{|c|c|c|c|c|}
\hline \multirow[b]{2}{*}{ Material } & \multirow[b]{2}{*}{ Test type } & \multicolumn{3}{|c|}{ Damage threshold (MPa) } \\
\hline & & $\begin{array}{l}\text { Strains } \\
\text { analysis }\end{array}$ & $\begin{array}{l}\text { Linear } \\
\text { analysis }\end{array}$ & $\begin{array}{c}\text { Nonlinear } \\
\text { analysis }\end{array}$ \\
\hline sandstone & uniaxial & 95 & 93 & 70 \\
\hline sandstone & uniaxial & 82 & 80 & 65 \\
\hline sandstone & uniaxial & 93 & 90 & 70 \\
\hline sandstone & $\begin{array}{c}\text { triaxial } \\
\left(P_{c}=5 \mathrm{MPa}\right)\end{array}$ & 170 & 165 & 135 \\
\hline sandstone & $\begin{array}{c}\text { triaxial } \\
\left(P_{c}=10 \mathrm{MPa}\right)\end{array}$ & 205 & 200 & 150 \\
\hline deep marl & uniaxial & 10 & 10 & 5 \\
\hline deep marl & uniaxial & 12 & 12 & 8 \\
\hline deep marl & $\begin{array}{c}\text { triaxial } \\
\left(P_{c}=5 \mathrm{MPa}\right)\end{array}$ & 20 & 18 & 15 \\
\hline granite & uniaxial & 80 & 80 & 60 \\
\hline granite & $\begin{array}{c}\text { triaxial } \\
\left(P_{c}=20 \mathrm{MPa}\right)\end{array}$ & 140 & 135 & 110 \\
\hline
\end{tabular}


standard method of strain measurements, the greater sensitivity of the nonlinear part allows forward detection of the damage threshold.

\section{REFERENCES}

Barrett, J. F. (1963), The Use of Functionals in the Analysis of Nonlinear Physical Systems, J. Elect. and Control, 567-615.

Berest, P., Bergues, J., Homand, F., Troalen, J. P., Henry, J. P., and Ikogon, S., Comportement thermique et mécanique du grés de Fontainebleau (Acute du col. Bilan et perspective du GRECO Géomatériaux 1989) pp. 19-52.

Boyd, S., TANG, Y. S., and ChuA, L. O. (1983), Measuring Volterra Kernels, IEEE Transaction on Circuits and Systems 30, 571-577.

BUI, H. D. (1993), Introduction aux problèmes inverses en mécanique des matériaux ( $\mathrm{n}^{\circ}$ 83) (EYROLLES 1993) pp. 75-90.

Choi, C. H., and Warren, M. E. (1978), Identification of Nonlinear Discrete Systems (Proc, South Western Conf. Atlanta 1978), 329-333.

GHOREYCHI, M. (1978), Mesure de l'endomagement des roches par l'attenuation des ultrasons (Proc. $2^{\text {ème }}$ colloque Franco-Polonais de géotechnique Nancy 1978) pp. 113-121.

Gladwin, M. T., and Sacey, F. D. (1974), Inelastic Degradation of Acoustic Pulses in Rocks, Ph. of Earth and Planetary Int. 8, North-Holland Pub. Co.

Heitz, J. F. (1992), Prepagation d'ondes en milieu non-linéaire (Thèse de doctorat Université J. Fourier Grenoble).

Hung, G., Stark, L., and Eykhoff, P. (1977), On the Interpretation of Kernels, Ann. Biomed. Eng. 5, $130-143$.

Liu, H. (1991), Multi-dimensional Signal Processing for Nonlinear Structural Dynamics, Mechan. Syst. and Signal Proc. 5, 61-80.

VINH, T. (1987), Techniques for the Identification of Nonlinearity (Course note Heriot-Watt University, Edinburgh 1987).

Wolters, R. (1971), Initial Fracturing in Rock under Triaxial Loading (Proc. Symp. of the Int. Soc. for Rock Mech., Nancy 1971), sec. 1.5. 